\title{
A theoretical study of chemical bonding and topological and electrostatic properties of the anti-leprosy drug dapsone
}

\author{
Niranjana Devi Rajendran ${ }^{3,4}$ - Natarajan Mookan ${ }^{1}$ (D) - Israel Samuel ${ }^{4} \cdot$ Sarath Babu Mookan $^{2}$ (D) \\ Govindarajan Munusamy $^{5} \cdot$ Selvaraj Gurudeeban $^{6} \cdot$ Satyavani Kaliamurthi $^{6}$
}

Received: 20 February 2020 / Accepted: 24 April 2020/Published online: 15 May 2020

(C) The Author(s) 2020

\begin{abstract}
The theoretical charge density study for the gas phase of anti-leprosy drug Dapsone has been carried out in the light of the theory of atoms in molecules using density functional theory employing B3LYP $(6-311 \mathrm{G}++(\mathrm{d}, \mathrm{p})$ hybrid functional completed with dispersion corrections. The Hirshfeld surface analysis as well as fingerprint plots has been utilized to visualize and quantify the intermolecular contacts present in the molecule. The topological properties such as electron density and its Laplacian, delocalization index have been elucidated to throw light into the chemical bonding and atomic and molecular details. The electron localization function has been used to visualize and deduce information on the lone pair and the subshells of the $\mathrm{Cl}$ atom. The electrostatic potential visualizes the positive and negative electrostatic potential regions which are susceptible to nucleophilic and electrophilic attack. On the whole, this study provides an exact mechanism, interaction, and topological and electrostatic properties of the drug through theoretical insights which all will be a platform for our further investigation of the interaction between dapsone and dihydropteroate synthase (DHPS).
\end{abstract}

Keywords Hirshfeld surface analysis · Quantum chemical calculations · Charge density analysis · Bond topological properties · Electrostatic properties

Natarajan Mookan

natarajanmkudu@mail.zjxu.edu.cn

Israel Samuel

israel.samuel@gmail.com

Sarath Babu Mookan

sarathbabum@gargi.du.ac.in

1 College of Biological, Chemical Sciences and Engineering, Jiaxing University, Jiaxing, Zhejiang 314001, China

2 Department of Chemistry, Gargi College, University of Delhi, New Delhi 110049, India

3 Research Centre of Physics, Fatima College, Madurai 625018, Tamil Nadu, China

4 Research and Postgraduate, Department of Physics, The American College, Madurai 625002, Tamil Nadu, India

5 Arignar Anna Government Arts and Science College, Nehru Nagar, Karaikal, Puducherry State, India

6 Center of Interdisciplinary Science-Computational Life Sciences, College of Food Science and Engineering, Henan University of Technology, Zhengzhou High-tech Industrial Development Zone, 100 Lianhua Street, Zhengzhou, Henan 450001, China

\section{Introduction}

Dapsone, an antibacterial, is primarily and effectively used in the leprosy treatment which is also used in combination with rifampicin and clofazimine for the treatment of Mycobacterium leprae infections (leprosy) [1-4], malaria [5-7], and pneumocystis pneumonia (PCP) [8-10]. Specifically, dapsone stops the bacterial dihydrofolic acid synthesis through the process of binding itself in the active site of the enzyme named 6-hydroxymethyl-7,8-dihydropteroate synthase (DHPS), which takes part in the condensation of para-aminobenzoic acid (pABA) with 6-hydroxymethyl-7,8dihydropterin-pyrophosphate to form 7,8-dihydropteroate and pyrophosphate [11]. Moreover, dapsone competes with paraaminobenzoate on the active site of DHPS and inhibits the bacterial dihydrofolic acid synthesis [12]. In present literature, Mendes et al. carried out the geometric and electronic study of dapsone and discussed the symmetric and asymmetric conformational isomer of the molecule [13]. Borges et al. carried out the first density functional study of the dapsone derivatives on methemoglobin [14]. Bhattacharya et al. revisited dapsone photophysics in a different solvent [15]. To the best of our 
knowledge, neither the study of theoretical charge density nor the topological properties for the gas phase of the molecule have been discussed in the literature.

The elaborate study on charge density in the light of AIM theory and electrostatic mapping of a molecule is very important due to their crucial application in determining the interaction of pharmaceutical compounds with a biomolecule. As referred to Bader the pioneer stated in AIM theory: "A Quantum theory [16], while the theory has its origin in quantum mechanics, its vehicle of expression, is the charge density". The charge density being the most important property is much useful in finding one-electron properties, ground-state properties of the molecules, type and the strength of the chemical bonding between the atoms, information on lattice energies, orbital locating and molecular interactions, etc. Especially hydrogen bonding serves as the principal source for the intermolecular interactions which further helps in tailoring more compounds with desired physical and chemical properties.

In the present work, an attempt has been made to reconstruct the charge density mapping of dapsone (4-[(4aminobenzene) sulfonyl] aniline) molecule using theoretical models and is thoroughly analyzed with the help of Quantum theory of atoms in molecules for the intuitive information on the molecule itself. In a theoretical model such as DFT (density functional theory) [17], a free dapsone molecule has been treated and analyzed for its electronic properties. The Hirshfeld surface analysis $[18,19]$ has been carried out for a clear understanding of the intermolecular hydrogen bonding interactions, and the fingerprint plot has been mapped for calculating the percentage of contribution of various bonds present in the molecule. The topology of the charge density has been analyzed, and the critical points in the charge density have been determined. The Lipinski rule of five has been calculated in order to appreciate the potential of the drug. Electrostatic potential (ESP) surfaces have been examined for the identification of not only the electrophilic and nucleophilic regions of the molecule but also understanding the lock and key mechanism. The results obtained from this study will be a manifesto for the further exploration of interpretation of the drug-receptor interactions between the dapsone molecule and the enzyme of dihydropteroate synthase (DHPS).

\section{Computational details}

The experimentally determined position values of the atoms of dapsone have been given as input for the theoretical optimization in GAUSSIAN09 software package [20] with DFT method using (B3LYP $\backslash 6-311 \mathrm{G}++(\mathrm{d}, \mathrm{p})$ ) level of theory [21, 22]. The single-point calculations have been performed once the convergence has been reached. The absence of imaginary frequencies has shown that the minimum energy structure has been achieved. The obtained wave function for the gas phase of the dapsone molecule has been given as an input to the AIM ALL package [23] for calculating the topological properties. The AIM-UC 4.0. [24] and MULTIWFN [25] software packages have been utilized for mapping purposes.

\section{Results and discussion}

\section{Description of structure}

The minimum energy structure of the dapsone molecule has been shown in Fig. 1a. In both the aniline rings, the standard values for $\mathrm{C}-\mathrm{C}$ and $\mathrm{C}-\mathrm{H}$ bonds are $1.39 \AA$ and $1.09 \AA$, and the values from our theoretical calculations lie in the range 1.387$1.406 \AA$ and $1.083-1.085 \AA$ which agrees well with the standard values [26]. The expected bond length for the $\mathrm{C}-\mathrm{N}$ bond is $1.48 \AA$, but the optimized bond lengths for the $\mathrm{C}-\mathrm{N}$ bond are in the range 1.387-1.388 $\AA$ where the decrease in bond length might be due to the resonance among $\mathrm{C}-\mathrm{N}$ bonds. The calculated angle for the plane O1-S1-O2 is found to be $120^{\circ}$ which suggests the trigonal planar geometry and sp2 hybridization state for the corresponding central $\mathrm{S} 1$ atom.

The shapes of the two amine groups are found to be a slight tetrahedral with the expected bond angle $113^{\circ}$. The atoms of $\mathrm{N} 1$ and $\mathrm{N} 2$ make equal dihedral angles with the respective planes $\mathrm{C} 3-\mathrm{C} 4-\mathrm{C} 5$ and $\mathrm{C} 9-\mathrm{C} 10-\mathrm{C} 11$ and are found to be

Fig. 1 a Optimized structure of dapsone. b View of the crystal packing in dapsone (along the $\mathbf{c}^{*}$ axis)

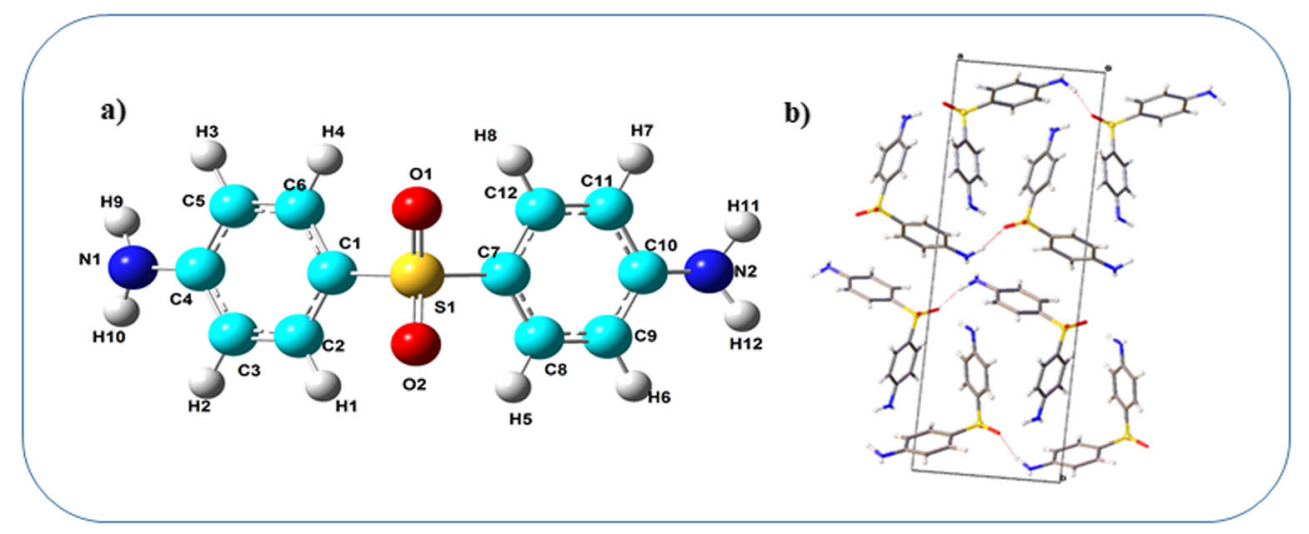




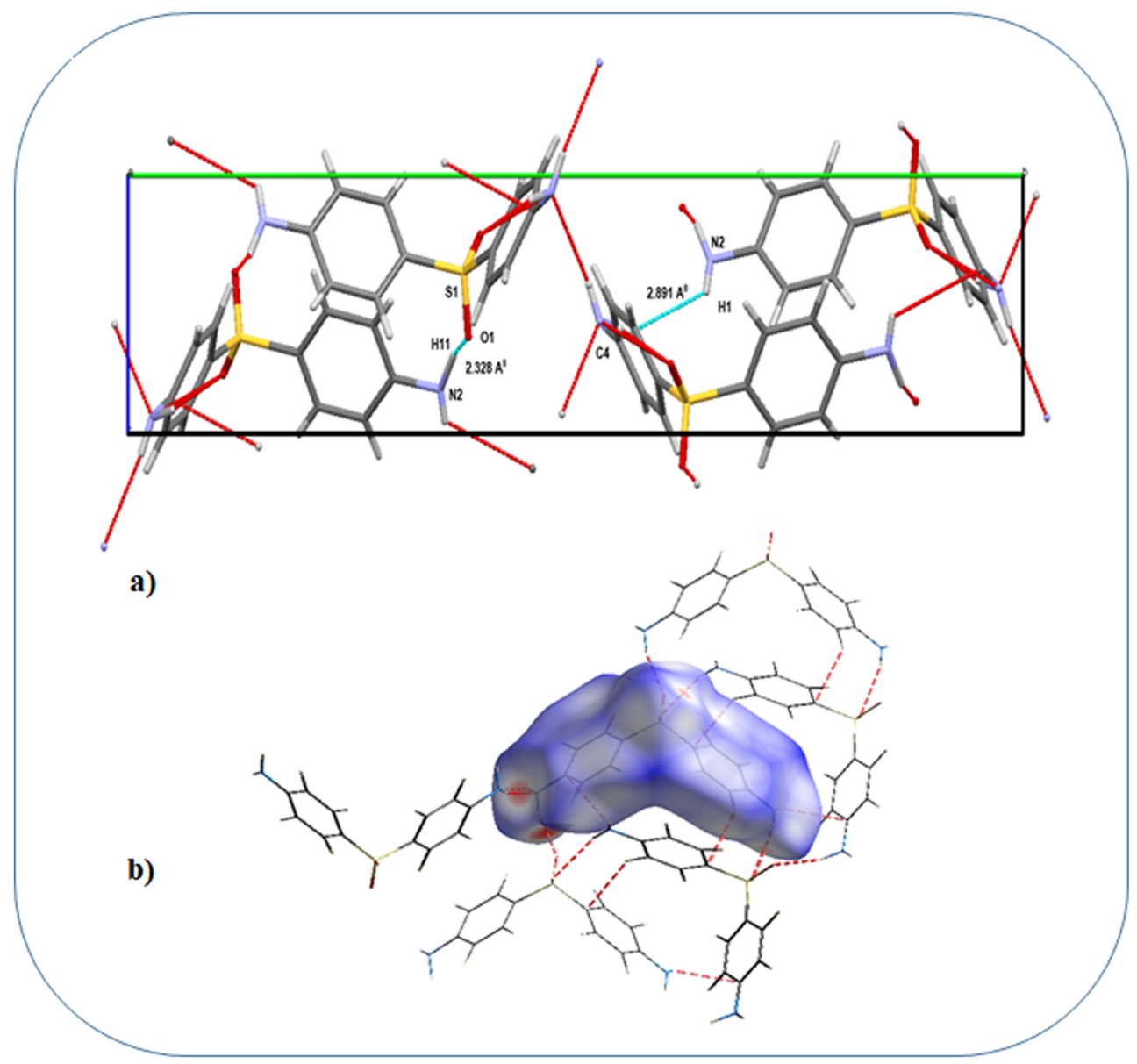

Fig. 2 a View of the intermolecular hydrogen bonds (along with a axis). b Visualization of the Hirshfeld surface of the strong interactions

$177^{\circ}$.The torsion angles which are formed by the atoms $\mathrm{C} 1$ and $\mathrm{C} 7$ with $\mathrm{O} 2-\mathrm{S} 1-\mathrm{O} 1$ plane individually are found to be $123.4^{\circ}$ which clearly suggest the symmetry of the molecule.

\section{Hydrogen bonding interaction}

In order to better understand the packing and interactions of the molecule with the neighboring molecules, the information from the experimental cif file has been given as the input for CrystalExplorer software 3.0 [18, 27]. The packing of the molecules in the crystal lattice (Fig. 1b) is viewed along $\mathbf{c}^{*}$ axis and the intermolecular interactions are sustained by Van der Waals interactions.

The molecular packing of the crystal is stabilized by $\mathrm{N}$ H...O, N-H...N, and $\pi . . \pi$ types of intermolecular interactions (Fig. 2) and is classified as strong and weak interactions with respect to their H...A distances. From Fig. 2a, it is obvious that there is no intramolecular $\mathrm{N}-\mathrm{H}$... O hydrogen bond formation, but two types of intermolecular $\mathrm{N}-\mathrm{H}$... O hydrogen bond formation is possible.

The hydrogen bond parameters of the strongest N1-H9... $\mathrm{O} 2$ interaction are: $\mathrm{H} 9 . . \mathrm{O} 2 ; 1.990 \AA$ and $\mathrm{N} 1-\mathrm{H} 9,1.009 \AA$, and the angle is $159.46^{\circ}$, and another weak N2-H11..O 1

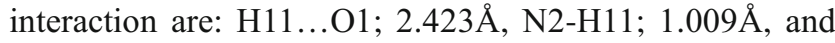
the angle is $145.29^{\circ}$. The hydrogen bonding parameters are given in Table 1. The existence of intermolecular hydrogen bonds between the molecules makes the interactions much stronger which would result in more stable and lower boiling points because the molecules prefer to be close vicinity with respect to each other. The stability of the dapsone molecule is also established by the high chemical hardness value (2.46a.u). Visualization of intermolecular interactions through the Hirshfeld surface analysis and obtaining the quantitative details of the contacts through the fingerprint analysis [28] are necessary to understand qualitatively and quantitatively the intermolecular interactions.

Table 1 Hydrogen bonding parameters (Åo)

\begin{tabular}{|c|c|c|c|c|}
\hline $\mathrm{D}-\mathrm{H} \ldots \mathrm{A}$ & D-H & Н...A & D...A & $\mathrm{D}-\mathrm{H} \ldots \mathrm{A}$ \\
\hline N1-H9...O2 & 1.009 & 1.990 & 2.956 & 159.46 \\
\hline N1-H10...N1 & 1.009 & 2.777 & 3.269 & 163.31 \\
\hline N2-H12 ...C9 & 1.009 & 2.743 & 3.369 & 120.47 \\
\hline N2-H11...O1 & 1.009 & 2.423 & 3.623 & 145.29 \\
\hline C11-H7 ...C7 & 1.083 & 2.728 & 3.303 & 139.76 \\
\hline
\end{tabular}


Fig. 3 Fingerprint plot a full and b resolved into $\mathrm{H}$....H contacts. c Percentage contribution of various intermolecular contacts in the molecule
The combination of $d i$ and $d e$ in the form of a 2D fingerprint plot is used to provide ample information regarding the percentage contribution of various intermolecular contacts present in the molecule. Figure $2 \mathrm{~b}$ shows thick orange color circular patches which represent the strong N1-H9... 2 2, N1$\mathrm{H} 10 \ldots \mathrm{N} 1$, and $\mathrm{N} 2-\mathrm{H} 11 \ldots \mathrm{O} 1$ hydrogen-bonding interactions which serve as an attestation for the strong intermolecular interactions. The fingerprint plot of the dapsone molecule (Fig. 3a) shows two sharp spikes at the bottom left and bottom right representing the donor and acceptor of $\mathrm{N}-\mathrm{H}$...O interaction. The distinct spike of the H...H type interaction is shown in Fig. 3b. Among the investigated interactions, H...H interaction has a major contribution $(36.4 \%)$, while the C...H interaction occupies $33.6 \%$ of the area of the total Hirshfeld surface. The various intermolecular contacts and their percentage contributions are shown in Fig. 3c. c)

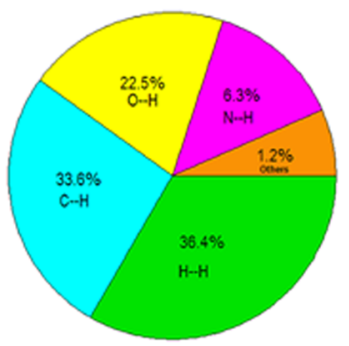

\section{Topological properties of electron density}

Investigation on topological properties of electron density in the light of AIM theory allows us to look into the details of atomic, molecular, and chemical bonding. Figure 4 a shows the static deformation density map of the top aniline ring where all the bonds exhibit covalent nature (Table 2). The closed-shell interactions between $\mathrm{S}-\mathrm{O}$ atoms are clearly visible in Fig. $4 \mathrm{~b}$.

The range of electron density and Laplacian of $\mathrm{C}-\mathrm{C}$ bonds in both the phenyl rings is $2.05 \mathrm{e} / \AA 3$ to $2.105 \mathrm{e} / \AA 3$ and $-20.1 \mathrm{e} /$ $\AA 5$ to $-20.8 \mathrm{e} / \AA \AA$ 5 . The $\mathrm{C}-\mathrm{S}$ bonds exhibit very poor electron density as well as Laplacian values which shows that the bonds are weak. Both the $\mathrm{C}-\mathrm{N}$ bonds show the same charge density and Laplacian values and are found to be $2.045 \mathrm{e} / \AA 3$ and $-21.25 \mathrm{e} / \AA \AA 5$, respectively, and this might be stabilized with same charge environment. Similarly, the $\mathrm{N}-\mathrm{H}$ and $\mathrm{C}-\mathrm{H}$
Fig. 4 Static deformation density map of the $\mathbf{a}$ top aniline ring and $\mathbf{b}$ $\mathrm{SO} 2$ group of the dapsone molecule

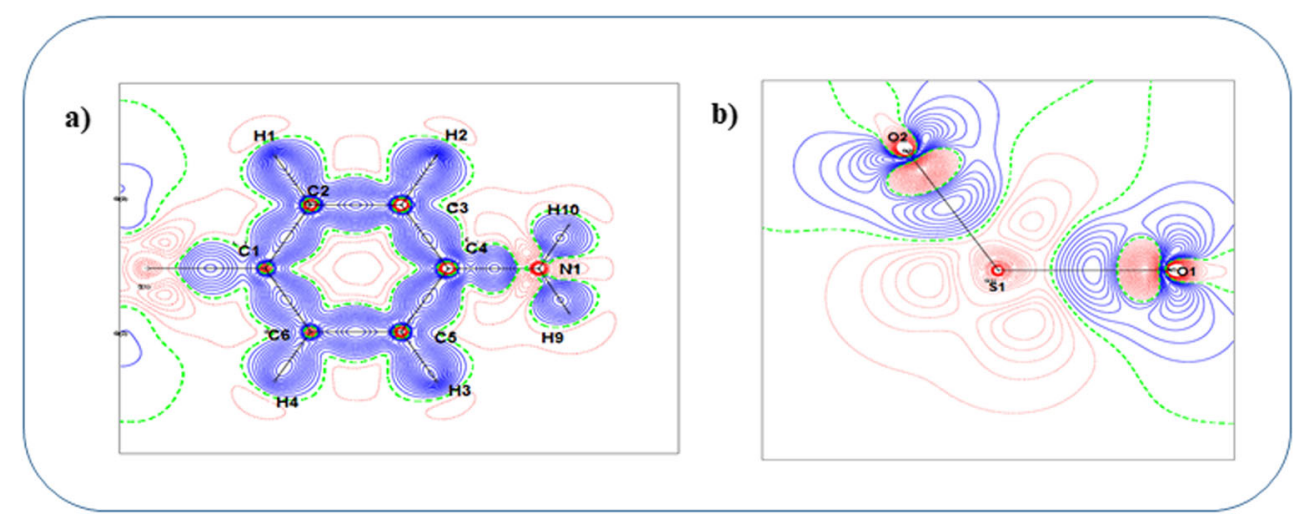


Table 2 Topological properties of the dapsone molecule

\begin{tabular}{|c|c|c|c|c|c|c|c|c|}
\hline Bonds & $\rho B C P(\mathrm{e} / \AA ̊ \AA 3)$ & $\nabla^{2} \rho(\mathrm{e} / \AA ̊ 5)$ & $\varepsilon$ & $V($ r) (a.u.) & $G($ r) (a.u.) & $K($ r) (a.u.) & $L(\mathrm{r})$ (a.u.) & DI \\
\hline $\mathrm{C} 1-\mathrm{C} 2$ & 2.06 & -20.2 & 0.20 & -0.411 & 0.101 & 0.310 & 0.209 & 1.34 \\
\hline $\mathrm{C} 2-\mathrm{C} 3$ & 2.10 & -20.8 & 0.22 & -0.425 & 0.104 & 0.320 & 0.216 & 1.42 \\
\hline $\mathrm{C} 3-\mathrm{C} 4$ & 2.05 & -20.1 & 0.21 & -0.401 & 0.096 & 0.305 & 0.209 & 1.28 \\
\hline $\mathrm{C} 4-\mathrm{C} 5$ & 2.05 & -20.1 & 0.21 & -0.401 & 0.096 & 0.305 & 0.209 & 1.28 \\
\hline C5-C6 & 2.10 & -20.8 & 0.22 & -0.425 & 0.104 & 0.320 & 0.216 & 1.34 \\
\hline $\mathrm{C} 1-\mathrm{C} 6$ & 2.06 & -20.2 & 0.20 & -0.411 & 0.101 & 0.310 & 0.209 & 1.28 \\
\hline C7-C8 & 2.06 & -20.2 & 0.20 & -0.411 & 0.101 & 0.310 & 0.209 & 1.34 \\
\hline C8-C9 & 2.10 & -20.8 & 0.22 & -0.425 & 0.104 & 0.320 & 0.216 & 1.42 \\
\hline C9-C10 & 2.05 & -20.1 & 0.21 & -0.401 & 0.096 & 0.305 & 0.209 & 0.86 \\
\hline $\mathrm{C} 10-\mathrm{C} 11$ & 2.05 & -20.1 & 0.21 & -0.402 & 0.096 & 0.305 & 0.209 & 1.29 \\
\hline $\mathrm{C} 11-\mathrm{C} 12$ & 2.10 & -20.8 & 0.22 & -0.425 & 0.104 & 0.320 & 0.216 & 1.34 \\
\hline $\mathrm{C} 7-\mathrm{C} 12$ & 2.06 & -20.2 & 0.20 & -0.411 & 0.101 & 0.310 & 0.209 & 1.29 \\
\hline $\mathrm{C} 1-\mathrm{S} 1$ & 1.36 & -9.14 & 0.09 & -0.190 & 0.048 & 0.142 & 0.095 & 0.86 \\
\hline C7-S1 & 1.36 & -9.12 & 0.09 & -0.189 & 0.048 & 0.142 & 0.094 & 1.42 \\
\hline $\mathrm{C} 4-\mathrm{N} 1$ & 2.04 & -21.2 & 0.07 & -0.570 & 0.175 & 0.395 & 0.219 & 1.07 \\
\hline $\mathrm{C} 10-\mathrm{N} 2$ & 2.04 & -21.2 & 0.07 & -0.567 & 0.174 & 0.393 & 0.219 & 1.07 \\
\hline C2-H1 & 1.92 & -23.9 & 0.01 & -0.319 & 0.035 & 0.283 & 0.248 & 1.42 \\
\hline C3-H2 & 1.88 & -22.9 & 0.03 & -0.318 & 0.040 & 0.277 & 0.237 & 0.93 \\
\hline C5-H3 & 1.88 & -22.9 & 0.03 & -0.318 & 0.040 & 0.277 & 0.237 & 0.96 \\
\hline C6-H4 & 1.92 & -23.9 & 0.01 & -0.319 & 0.035 & 0.283 & 0.248 & 0.84 \\
\hline C8-H5 & 1.92 & -23.9 & 0.01 & -0.319 & 0.035 & 0.283 & 0.248 & 0.93 \\
\hline C9-H6 & 1.88 & -22.9 & 0.03 & -0.318 & 0.040 & 0.277 & 0.237 & 0.96 \\
\hline C11-H7 & 1.88 & -22.9 & 0.03 & -0.318 & 0.040 & 0.277 & 0.237 & 0.96 \\
\hline C12-H8 & 1.92 & -23.9 & 0.01 & -0.319 & 0.035 & 0.283 & 0.248 & 0.93 \\
\hline $\mathrm{S} 1-\mathrm{O} 1$ & 1.93 & 20.3 & 0.01 & -0.899 & 0.555 & 0.344 & -0.210 & 1.19 \\
\hline $\mathrm{S} 1-\mathrm{O} 2$ & 1.93 & 20.3 & 0.01 & -0.899 & 0.555 & 0.344 & -0.210 & 1.19 \\
\hline N1-H9 & 2.30 & -38.5 & 0.06 & -0.513 & 0.057 & 0.456 & 0.399 & 0.84 \\
\hline N1-H10 & 2.30 & -38.5 & 0.06 & -0.513 & 0.057 & 0.456 & 0.399 & 0.96 \\
\hline N2-H11 & 2.30 & -38.5 & 0.06 & -0.512 & 0.057 & 0.456 & 0.399 & 0.84 \\
\hline N2-H12 & 2.30 & -38.5 & 0.06 & -0.512 & 0.057 & 0.456 & 0.399 & 0.84 \\
\hline
\end{tabular}

bonds show good accumulation of charge density between the bonds (Fig. 5a). In addition to the electron density, the Laplacian and electron localization function also reveal the closed-shell interacting nature of the $\mathrm{S}-\mathrm{O}$ bonds with the positive Laplacian value $(20.35 \mathrm{e} / \AA 5$ ) as well as through the plots of Figs. $5 \mathrm{~b}$ and 6 . The lone pairs of $\mathrm{O}$ atoms and the $\mathrm{K}$, L, and $\mathrm{M}$ shells of $\mathrm{S}$ atom are also clearly visible in Fig. 6. The molecular graph with the bond as well as ring critical point has been shown in Fig. 7.
Fig. 5 Laplacian of the electron density of a top aniline ring and $\mathbf{b}$ $\mathrm{SO} 2$ group of the dapsone molecule

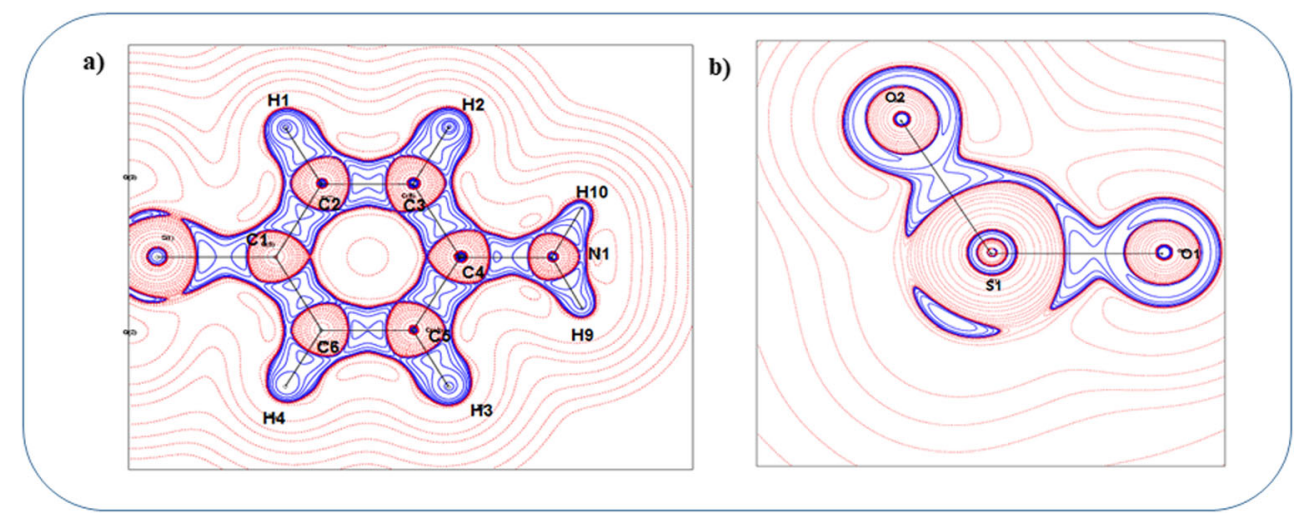




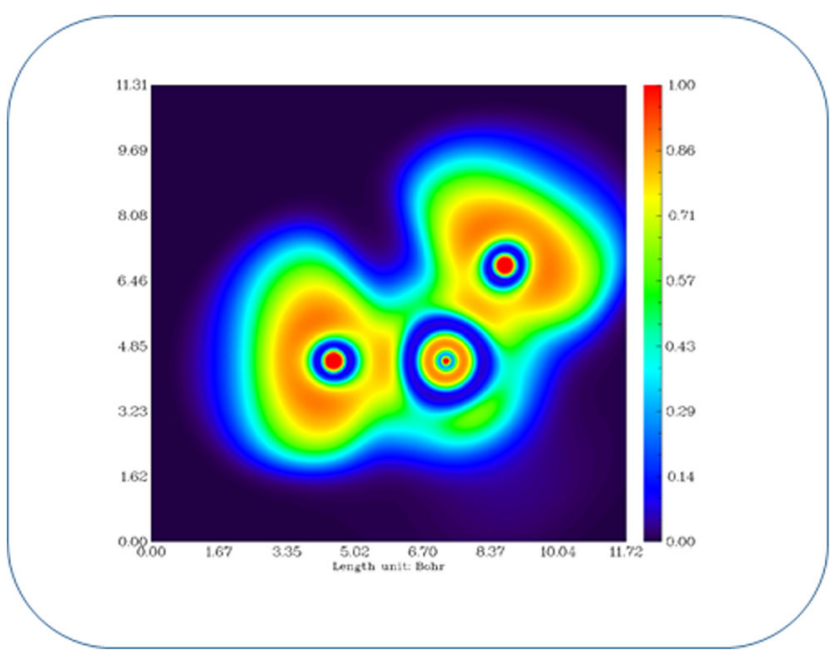

Fig. 6 Visualization of electron localization function

The very low ellipticity value (ca 0.01 ) for the bonds $\mathrm{S}-\mathrm{O}$ and $\mathrm{C}-\mathrm{H}$ portrays the near cylindrically symmetric nature. The ellipticity values for the $\mathrm{C}-\mathrm{C}$ bonds are in the range $0.20-0.22$, and the increased values show the $\pi$ character of the $\mathrm{C}-\mathrm{C}$ bonds.

Among the $\mathrm{C}-\mathrm{C}$ bonds, the bonds $\mathrm{C} 2-\mathrm{C} 3$ and $\mathrm{C} 8-\mathrm{C} 9$ have the highest delocalization index value which shows not only the strength of the bonds but also denotes the occurrence of resonance/delocalization of the bonds in both the phenyl rings. Among the $\mathrm{C}-\mathrm{H}$ bonds, the $\mathrm{C} 2-\mathrm{H} 1$ bond has the highest delocalization index value which is supported by the high charge density values $(1.92 \mathrm{e} / \AA 3)$ as well as high Laplacian values $(-23.9 \mathrm{e} / \AA \mathbf{5} 5)$. Similarly, the C7-S1 bond exhibits a high value of the delocalization index (1.42) even though the charge density values of both the $\mathrm{C}-\mathrm{S}$ bonds are the same. This might be because of the influence of the $\mathrm{S} 1$ atom that is higher in $\mathrm{C} 7-\mathrm{S} 1$ bond than the $\mathrm{C} 1-\mathrm{S} 1$ bond.

As the kinetic energy density dominates, the total energy density becomes positive which shows the instability of the bonds. Among all the bonds, the $\mathrm{N}-\mathrm{H}$ bonds have high total energy density values which show the low stability of the bonds.

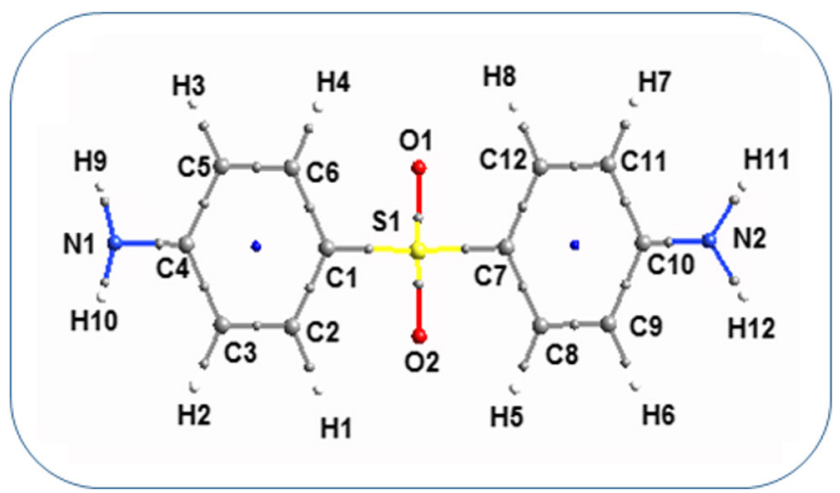

Fig. 7 Molecular graph diagram with a bond (gray small circles) and ring (blue small circles) critical points of dapsone molecule

Table 3 Bader charges and atomic volumes

\begin{tabular}{|c|c|c|c|c|c|}
\hline Atoms & $\mathrm{Q}(\mathrm{e})$ & $\mathrm{V}(\AA 3)$ & Atoms & $\mathrm{Q}(\mathrm{e})$ & $\mathrm{V}(\AA 3)$ \\
\hline S1 & 2.364 & 54.74 & $\mathrm{C} 11$ & -0.011 & 72.05 \\
\hline $\mathrm{O} 1$ & -1.267 & 111.18 & $\mathrm{C} 12$ & 0.014 & 69.36 \\
\hline $\mathrm{O} 2$ & -1.267 & 111.19 & H1 & 0.064 & 35.25 \\
\hline N1 & -1.074 & 97.69 & $\mathrm{H} 2$ & 0.016 & 37.58 \\
\hline $\mathrm{N} 2$ & -1.072 & 97.67 & $\mathrm{H} 3$ & 0.016 & 37.59 \\
\hline $\mathrm{C} 1$ & -0.155 & 68.12 & $\mathrm{H} 4$ & 0.064 & 35.26 \\
\hline $\mathrm{C} 2$ & 0.014 & 69.36 & H5 & 0.064 & 35.23 \\
\hline $\mathrm{C} 3$ & -0.011 & 71.99 & H6 & 0.016 & 37.62 \\
\hline $\mathrm{C} 4$ & 0.404 & 54.69 & $\mathrm{H} 7$ & 0.016 & 37.62 \\
\hline $\mathrm{C} 5$ & -0.011 & 72.01 & H8 & 0.064 & 35.22 \\
\hline C6 & 0.014 & 69.36 & H9 & 0.374 & 23.84 \\
\hline C7 & -0.155 & 68.09 & H10 & 0.374 & 23.83 \\
\hline $\mathrm{C} 8$ & 0.014 & 69.33 & H11 & 0.373 & 23.86 \\
\hline C9 & -0.011 & 72.02 & $\mathrm{H} 12$ & 0.373 & 23.86 \\
\hline $\mathrm{C} 10$ & 0.402 & 54.68 & & & \\
\hline
\end{tabular}

\section{Atomic charges and volume}

The sulfur atom exhibits a very high positive Bader atomic charge (2.36e) as it is attached with the two most electronegative oxygen atoms $(-1.27 \mathrm{e})$ on both sides. The electronegative N1 and $\mathrm{N} 2$ atoms exhibit negative charges $(-1.07 \mathrm{e})$, and they are attached with the two positive $\mathrm{H}$ atoms each. The group charge of both the primary amine (-NH2) is negative $-0.23 \mathrm{e})$, and they can donate electrons as donors. Both the $\mathrm{C}-\mathrm{N}$ bonds are well polarized where the carbon atoms $(\mathrm{C} 4, \mathrm{C} 10)$ possess positive charges $(0.40 \mathrm{e})$ and the nitrogen atoms $(\mathrm{N} 1, \mathrm{~N} 2)$ possess negative charges $(-1.07 \mathrm{e})$. The most electronegative atoms $\mathrm{O} 1$ and $\mathrm{O} 2$ exhibit greater volume, and the values are found to be $111.18 \AA 3$ and $111.19 \AA 3$ respectively (Table 3 ).

\section{Physiochemical properties: PASS analysis}

Some of the molecular properties like membrane permeability and bioavailability are straightly connected with some molecular descriptors such as $\log \mathrm{P}$ (partition coefficient), molecular weight (MW), hydrogen bond acceptors, and donors count in a molecule. Lipinski rule of five [29] which is also used as a

Table 4 The physiochemical properties of the dapsone molecule

\begin{tabular}{lc}
\multicolumn{2}{l}{ Lipinski rule of 5 parameters } \\
\hline 1. mi Log P & -0.70 \\
2. TPSA & 86.19 \\
3. MW & 260.4 \\
4. HBA & 4 \\
5. HBD & 4 \\
6. No. of violations & 0 \\
7. No. of rotatable bonds & 2
\end{tabular}


Fig. 8 a The isosurface representation of the molecular electrostatic potential of the dapsone molecule. b Lock and key type structure with gridlines of the dapsone molecule

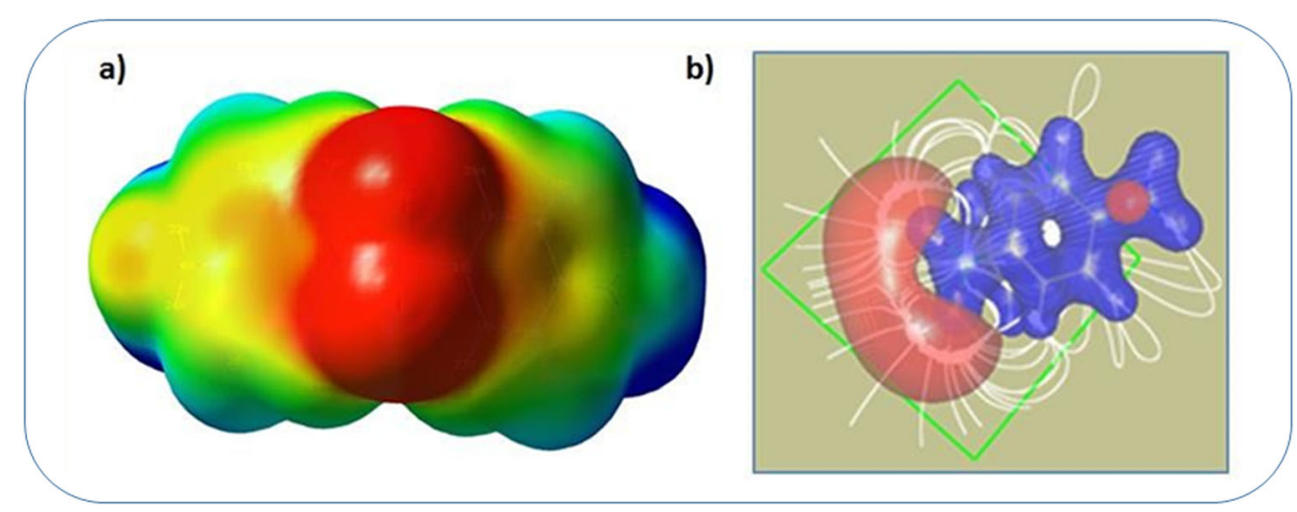

filter for drug-like properties says that most molecules with good membrane permeability have $\log \mathrm{P} \leq 5$, molecular weight $\leq 500$, number of hydrogen bond donors $\leq 5$, and number of hydrogen bond acceptors $\leq 5$. Lipinski rule of 5 parameters has been listed in Table 4. The compound satisfies the above conditions and proves itself as a potent drug and physiologically active.

\section{Electrostatic potential}

The electrostatic potential is primarily used to trace out the electrostatic positive as well as electrostatic negative regions where the ligand-receptor interactions possibly occur [30]. An asymmetric potential is obtained for the dapsone molecule, and the mapped electrostatic potential surfaces are shown in Fig. 8a where the negative region of the molecule seems to concentrate mainly on $\mathrm{O} 2-\mathrm{S} 1-\mathrm{O} 1$ (Fig. 8b), and it envelopes mainly on the oxygen atoms $\mathrm{O} 1$ and $\mathrm{O} 2$ as their respective negative charges are $-1.27 \mathrm{e}$ and the average bond length (S1$\mathrm{O} 1, \mathrm{~S} 1-\mathrm{O} 2)$ is $1.47 \AA$. The color gradient from blue to red shows the positive and negative regions of ESP which pronounces the electrophilic and nucleophilic regions of the molecule. The enveloping of negative nucleophilic to the positive

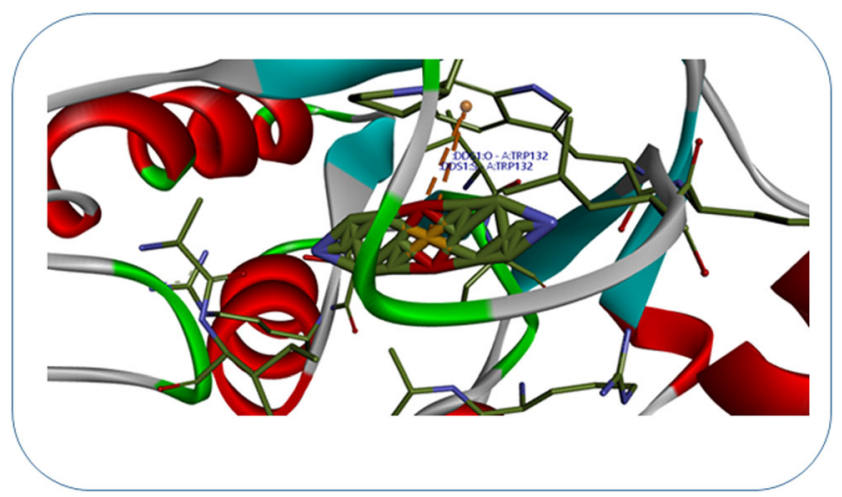

Fig. 9 Non-covalent $\pi$-cation interactions of dihydropteroate synthase (DHPS) and dapsone. The interacting residues were denoted in blue in color electrophilic seems to enact the lock and key structure as observed in protein-ligand interaction [31]. Figure $8 \mathrm{~b}$ shows the negative region (red) surrounding $\mathrm{O} 1$ and $\mathrm{O} 2$, whereas the positive regions (blue) of the molecule show the complete circuits of electric field gridlines, thus distinguishing electrophilic and nucleophilic regions. The grid lines showing the electric field are drawn for the bottom phenyl ring (Fig. 8b) at the isosurface value level 0.24 (blue) to -0.038 (red) which show the origin of the field grid lines centered at the negative envelope surrounding the atoms $\mathrm{O} 1$ and $\mathrm{O} 2$ and end up with the positive hydrogen ions.

As shown in the lock and key shape of the molecular electrostatic potential of dapsone, the negative potential which is in the vicinity of $\mathrm{O} 1$ and $\mathrm{O} 2$ atoms is susceptible to severe electrophilic attack. When dapsone is taken as an antibacterial, the nucleophilic/electrophilic region of dapsone interacts with the electrophilic/nucleophilic region of the dihydrofolic acid, and the electrophilicity of the new molecule gets equalized by the electronic charge transfer between the ligand and the receptor [32]. In this manner, dapsone inhibits the growth of a bacterial cell by discontinuing dihydrofolic acid synthesis.

\section{Molecular interaction of dihydropteroate synthase (DHPS) and dapsone}

\section{Homology modeling}

The crystal structure of DHPS is not available in protein database. Therefore, the proteomic sequence of DHPS (Uniprot ID: P0C0X1) of Mycobacterium leprae was obtained from Uniprot database. The homology modeling of extracted protein sequence was given as an input on the automated SWISSMODEL server [33]. Based on the integrated deep view method in the server, the complex crystal structure of DHPS of Mycobacterium tuberculosis was selected as a template (PDB ID: 1EYE). Finally, the refined structure was used for further studies. 


\section{Ligand preparation}

Two-dimensional structure of dapsone (CID: 2955) was obtained from PubChem database. The .sdf file converted into .pdb by using Open Babel tool [34].

\section{Molecular docking}

The molecular docking was performed by using Molecular Graphics Laboratory AutoDock (4.2) tools. The preparation of receptor and ligand, grid, and docking calculation was described in the previous studies [35].

\section{Molecular interaction of DHPS and dapsone}

Molecular docking calculation of dapsone with target enzyme dihydropteroate synthase (DHPS) generated six clusters of conformers using a root mean square difference (RMSD) tolerance of $2.0^{\circ} \mathrm{A}$. The two non-covalent $\pi$-cation interactions estimated on the amino acid residues TRP 132 of DHPS with respective oxygen and sulfur atoms of dapsone (Fig. 9). The results suggested that dapsone have played an important role in the dihydrofolic acid synthesis.

\section{Conclusions}

We have determined the theoretical charge density distribution for the gas phase of dapsone on the basis of the AIM theory. The identification of close contacts and distinguishing them from each other has been accomplished through the Hirshfeld surface analysis and the fingerprint plots. The topological analysis reveals the strength and chemical bonding details of all the covalent bonds in the molecule. The subshells of $\mathrm{S}$ atom and lone pairs of $\mathrm{O}$ and $\mathrm{N}$ atoms are visible in the ELF plots, and the ELF clearly shows the closed-shell interaction of the $\mathrm{S}-\mathrm{O}$ bonds. In the ESP map, a high electronegative region is found in the vicinity of the $\mathrm{O} 1$ and $\mathrm{O} 2$ atoms, and this is the active site for electrophilic attack. This theoretical charge density study clearly provides the fine details of structural information, intermolecular interaction, and charge density distribution which are the necessary parameters to interpret the drug-receptor interactions between the dapsone molecule and enzyme of dihydropteroate synthase (DHPS).

Acknowledgments The authors are grateful to the Principal \& Secretary and the Head of PG Department of Physics, The American College, Madurai, India, for their continued encouragement and support during the progress of this work.

Funding information The authors (S.I, R.N) are grateful for the grant provided by the University Grants Commission, India, in the form of Major Research Project (F.No. 41-848/2012(SR)).

\section{Compliance with ethical standards}

Conflict of interest The authors declare that they have no conflicts of interest.

Open Access This article is licensed under a Creative Commons Attribution 4.0 International License, which permits use, sharing, adaptation, distribution and reproduction in any medium or format, as long as you give appropriate credit to the original author(s) and the source, provide a link to the Creative Commons licence, and indicate if changes were made. The images or other third party material in this article are included in the article's Creative Commons licence, unless indicated otherwise in a credit line to the material. If material is not included in the article's Creative Commons licence and your intended use is not permitted by statutory regulation or exceeds the permitted use, you will need to obtain permission directly from the copyright holder. To view a copy of this licence, visit http://creativecommons.org/licenses/by/4.0/.

\section{References}

1. McEvoy GK (2005) Drug information resources and medication safety. In: Manasse Jr HR, Thompson KK (eds) Medication safety: a guide for health care facilities. American Society of HealthSystem Pharmacists, Bethesda, MD, pp 253-274

2. Shepard CC (1960) The experimental disease that follows the injection of human leprosy bacilli into foot-pads of mice. J Exp Med 112:445-454. https://doi.org/10.1084/jem.112.3.445

3. Waters MFR (1983) New approaches to chemotherapy for leprosy. Drugs 26:465-467. https://doi.org/10.2165/00003495-19832606000001

4. Wozel G, Blasum C (2014) Dapsone in dermatology and beyond. Arch Dermatol Res 306:103-124. https://doi.org/10.1007/s00403013-1409-7

5. Premji Z et al (2009) Chlorproguanil- dapsone- artesunate versus artemether- lumefantrine: a randomized, double-blind phase III trial in African children and adolescents with uncomplicated plasmodium falciparum malaria. PLoS One 4:e6682. https://doi.org/10. 1371/journal.pone.0006682

6. Tiono AB et al (2009) Chlorproguanil-dapsone-artesunate versus chlorproguanil-dapsone: a randomized, double-blind, phase III trial in African children, adolescents, and adults with uncomplicated plasmodium falciparum malaria. Am J Trop Med Hyg 81:969 978. https://doi.org/10.4269/ajtmh.2009.09-0351

7. Won YJ, Kim OL, Yu ST, Yoon YW, Choi DY (2007) A case of dapsone syndrome. Korean J Pediatr 50:493-496 https://www.kjp. or.kr/upload/pdf/2007500514-20070513110713.PDF

8. Castro JG, Morrison-Bryant M (2010) Management of Pneumocystis jirovecii pneumonia in HIV infected patients: current options, challenges and future directions. HIV/AIDS (Auckl) 2: 123-134. https://doi.org/10.2147/hiv.s7720

9. Hughes WT (1998) Use of dapsone in the prevention and treatment of Pneumocystis carinii pneumonia. Clin Infect Dis 27:191-204. https://doi.org/10.1086/514626

10. Lee BL, Ileana M, Benowitz NL, Peyton J, Wofsy CB, John MV (1989) Dapsone, trimethoprim, and sulfamethoxazole plasma levels during treatment of Pneumocystis pneumoniain patients with the acquired immunodeficiency syndrome (AIDS): evidence of drug interactions. Ann Intern Med 110:606-611. https://doi.org/10. 7326/0003-4819-110-8-606

11. Zhu YI, Stiller MJ (2001) Dapsone and sulfones in dermatology: overview and update. J Am Acad Dermatol 45:420-434. https://doi. org/10.1067/mjd.2001.114733 
12. Chaitanya S, Das M, Bhat P, Ebenezer M (2015) Computational modelling of dapsone interaction with dihydropteroate synthase in Mycobacterium leprae; insights into molecular basis of dapsone resistance in leprosy. J Cell Biochem 116:2293-2303. https://doi. org $/ 10.1002 /$ jcb. 25180

13. Anna Mendes PS, Schalcher TR, Barros TG, Almeida ED, Maia CSF, Barros CAL, Monteiro MC, Borges RS (2011). J Comput Theor Nanosci 8(4):1428-1431. https://doi.org/10.1166/jctn.2011. 1832

14. Borges RS, Romero OAS (2011) Structure and reactivity of Imidazolin-Azabutadienes. J Comput Theor Nanosci 8:11291131(3). https://doi.org/10.1166/jctn.2011.1789

15. Bhattacharya P, Sahoo D, Chakravorti S (2012) Revisit of 4, 4'diaminodiphenyl sulfone photophysics in different solvents. Ind Eng Chem Res 51:2505-2514. https://doi.org/10.1021/ie201113b

16. Salvador P, Ramos-Cordoba E (2013) Communication: an approximation to Bader's topological atom. J Chem Phys 139(7):071103. https://doi.org/10.1063/1.4818751

17. Parr RG (1980) Density functional theory of atoms and molecules. In: Horizons of Quantum Chemistry. Springer, pp 5-15. https://doi. org/10.1007/978-94-009-9027-2 2

18. McKinnon JJ, Spackman MA, Mitchell AS (2004) Novel tools for visualizing and exploring intermolecular interactions in molecular crystals. Acta Crystallogr Sect B: Struct Sci B60:627-668. https:// doi.org/10.1107/S0108768104020300

19. Spackman MA, Byrom PG (1997) A novel definition of a molecule in a crystal. Chem Phys Lett 267:215-220. https://doi.org/10.1016/ S0009-2614(97)00100-0

20. Frisch MJ, Trucks GW, Schlegel HB, Scuseria GE, Robb MA, Cheeseman JR et al (2009) Gaussian 09, revision A.02. Gaussian Inc, Wallingford http://www.gaussian.com

21. Becke AD (1992) Density-functional thermochemistry. I. The effect of the exchange-only gradient correction. J Chem Phys 96:21552160. https://doi.org/10.1063/1.462066

22. Lee C, Weitao Y, Parr RG (1988) Development of the Colle-Salvetti correlation-energy formula into a functional of the electron density. Phys Rev B 37:785-789. https://doi.org/10.1103/PhysRevB.37.785

23. Keith TA, Gristmill TK (2017) AIMAll (version 17.01.25) software, Overland Park, KS

24. Vega AD (2014) AIM-UC: an application for QTAIM analysis. J Comp Methods in Sci Eng 14:1-3. https://doi.org/10.3233/JCM140491

25. Lu T, Chen F (2012) Multiwfn: A multifunctional wavefunction analyzer. J Comput Chem 33:580-592. https://doi.org/10.1002/ jcc. 22885
26. Allen FH, Kennard O, Watson DG, Brammer L, Orpen AG, Taylor R (1987) Tables of bond lengths determined by X-ray and neutron diffraction. Part 1. Bond lengths in organic compounds. J Chem Soc Perkin Trans 2:S1-S19. https://doi.org/10.1039/P298700000S1

27. Wolff SK, Grimwood, DJ, McKinnon JJ, Turner MJ, Jayatilaka D, Spackman MA (2012) Crystal Explorer 3.0. University of Western Australia, Perth

28. Spackman MA, McKinnon JJ (2002) Fingerprinting intermolecular interactions in molecular crystals. CrystEngComm 4:378-392. https://doi.org/10.1039/B203191B

29. Lipinski CA, Lombardo F, Dominy BW, Feeney PJ (2001) Experimental and computational approaches to estimate solubility and permeability in drug discovery and development settings1PII of original article: S0169-409X(96)00423-1. The article was originally published in Adv Drug Deliv Rev 23(1997)3-25. https://doi. org/10.1016/S0169-409X(96)00423-1. Adv Drug Deliv Rev 46:326 https://doi.org/10.1016/S0169-409X(00)00129-0

30. Politzer P, Murray JS (2001) Computational prediction of condensed phase properties from statistical characterization of molecular surface electrostatic potentials. Fluid Phase Equilib 185:129137. https://doi.org/10.1016/S0378-3812(01)00463-0

31. Politzer P, Murray JS (2002) The fundamental nature and role of the electrostatic potential in atoms and molecules. Theor Chem Accounts 108:134-142. https://doi.org/10.1007/s00214-002-03639

32. Hans-Joachim Böhm, Schneider G (2003) Protein-ligand interactions: from molecular recognition to drug design. https://doi.org/10. $1002 / 3527601813$

33. Waterhouse A, Bertoni M, Bienert S, Studer G, Tauriello G, Gumienny R, Heer FT, de Beer TAP, Rempfer C, Bordoli L, Lepore R, Schwede T (2018) SWISS-MODEL: homology modeling of protein structures and complexes. Nucleic Acids Res 46(W1):W296-W303. https://doi.org/10.1093/nar/gky427

34. O'Boyle NM, Banck M, James CA, Morley C, Vandermeersch T, Hutchison GR (2011) Open babel: an open chemical toolbox. J Chem 3(1): 33. https://doi.org/10.1186/1758-2946-3-33

35. Selvaraj G, Kaliamurthi S, Thirugnasambandan R (2016) Effect of Glycosin alkaloid from Rhizophora apiculata in non-insulin dependent diabetic rats and its mechanism of action: in vivo and in silico studies. Phytomedicine 23(6):632-640. https://doi.org/10.1016/j. phymed.2016.03.004

Publisher's note Springer Nature remains neutral with regard to jurisdictional claims in published maps and institutional affiliations. 